\title{
New molecular semi-quantification tool provides reliable microbiological evidence for pulmonary infection
}

\author{
Javier Yugueros-Marcos ${ }^{1,2}$, Olivier Barraud ${ }^{3}$, Alexandra lannello ${ }^{1}$, Marie Cécile Ploy ${ }^{3}$, Christine Ginocchio ${ }^{4,5}$, \\ Margarita Rogatcheva ${ }^{4}$, Cristina Alberti-Seguii ${ }^{2}$, Alexandre Pachot ${ }^{1}$, Virginie Moucadel ${ }^{1}$, Bruno François B, $^{3,6} 7^{*}$ (i) \\ and For the VALIBI study group
}

(c) 2018 Springer-Verlag GmbH Germany, part of Springer Nature and ESICM

Dear Editor,

Ventilator-associated pneumonia (VAP) remains one of the most commonly encountered hospital-acquired infections worldwide, despite recent national surveillance data suggesting its declining incidence [1]. Many reports have been published on the use of large multiplex rapid diagnostic methods for various community-acquired infections, but their validity for the microbiological diagnosis of VAP using a semi-quantitative approach has not yet been assessed [2, 3].

Bronchoalveolar lavage (BAL) specimens from a prospective, non-randomized, non-interventional, multicenter clinical trial enrolling 120 patients [4] were used to evaluate the new BioFire ${ }^{\circledR}$ Pneumonia Panel (BPP). A total of 117 BAL specimens were tested with the BPP, which enabled the detection of 18 bacteria, 8 viruses, and 7 antimicrobial resistance-associated genes in $1 \mathrm{~h}$. Results were compared with conventional culture methods (CC). Assessment of concordance for the identification of 15 commonly detected bacteria was done, according to an agreed semi-quantitative threshold to report a positive result [5]. Clinicians were left blinded to the rapid test results and patients were treated according to local guidelines.

We found that the most frequent bacterial species in BAL were Staphylococcus aureus, Pseudomonas aeruginosa, and Haemophilus influenzae with 14 (12\%) of the

\footnotetext{
*Correspondence: b.francois@unilim.fr

${ }^{3}$ Univ. Limoges, Inserm, CHU Limoges, RESINFIT, U1092, Limoges, France

Full author information is available at the end of the article
}

specimens positive for the $c t x-m$ gene and $12(10 \%)$ for viruses. Overall bacterial identification concordance with CC was $89.0 \%$ positive percentage agreement (PPA) and 95.9\% negative percentage agreement (NPA). A total of 74 (39\%) identification calls were discrepant between both techniques. In 26 (14\%) cases, the same microorganism was detected by both BPP and CC, even though they were considered as apparent discrepancies, as a result of the different positivity threshold call used for each technique. Eleven (6\%) were false positive BPP calls that could be explained by an active antimicrobial therapy ongoing at the time of BAL collection. Overall concordance increases to 93.1\% PPA and 98.2\% NPA (Fig. 1) when reconsidering these discrepancies as true positive results. Additional investigations were carried out on the $10(5.2 \%)$ false negative identification calls remaining. While $4(2.0 \%)$ were linked to a wrong identification provided by CC, 6 (3.1\%) remained unexplained. This latter number appears acceptable when considering overall usual microbiological documentation in VAP with CC methods. If the molecular method was used to choose initial therapy, in 28 (29.1\%) patients, initial antimicrobial treatment should have been larger, as at least one microorganism reported by BPP was not covered; and in 16 (16.7\%) patients, initial antimicrobial therapy could have been more targeted, as prescription of at least one antimicrobial agent would not have been supported by the $\mathrm{BPP}$ results.

Our study is the first comparing concordance of a multiplexed molecular method with conventional culture, based on the use of specific semi-quantitative thresholds for positivity calls, enrolling more than 100 homogeneous 


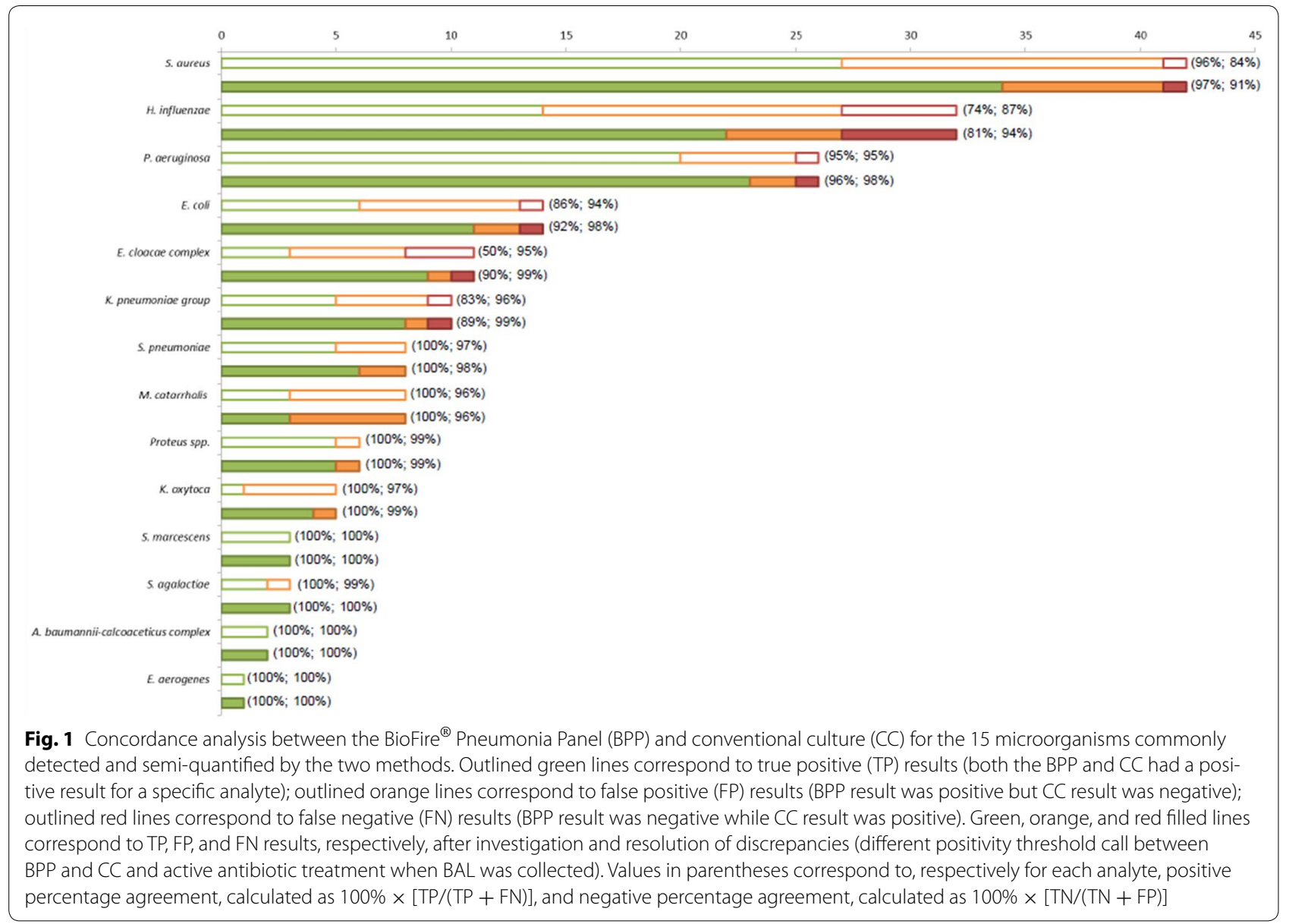

patients with suspected VAP. The availability of rapid reliable microbiological results and resistance information can be of major utility to effectively manage critically ill patients in the ICU with suspected or confirmed VAP but more comprehensive prospective studies are required to understand if the use of the BPP would have an overall positive impact in the management of VAP, including effect on antimicrobial use, clinical outcomes, and emergence of resistance even when considering some false negative results.

\section{Author details}

${ }^{1}$ Medical Diagnostic Discovery Department (MD3), bioMérieux S.A., Grenoble, France. ${ }^{2}$ bioMérieux S.A./BioFire LLC Research and Development, Grenoble, France. ${ }^{3}$ Univ. Limoges, Inserm, CHU Limoges, RESINFIT, U1092, Limoges, France. ${ }^{4}$ BioFire Diagnostics, LLC, Salt Lake City, UT, USA. ${ }^{5}$ bioMérieux Inc., Durham, NC, USA. ${ }^{6}$ Réanimation Polyvalente, CHU Dupuytren, Limoges, France. ${ }^{7}$ Inserm CIC1435, CHU Dupuytren, Limoges, France.

\section{Acknowledgements}

The authors gratefully acknowledge the VALIBI study group for their contribution to the VALIBI clinical study. The authors thank Isabelle Herafa from Inserm CIC 1435, Marc Clavel from CHU Limoges, and Marie-Astrid Jestin from bioMérieux for managing operations during the whole clinical study. The authors would like to thank Isabelle Sothier, Coralie Heyd, Caroline Dubost, Christelle Weber, and Rehane Ottaviani for their invaluable technical support running samples and carrying out investigations into discrepancies. The authors would like to thank Julien Textoris and Mark Miller for their thorough manuscript review and help in summarizing observations from this study. Funding was provided by bioMérieux S.A. and was part of ADNA (Advanced Diagnostic for New Therapeutic Approaches), a program dedicated to personalized medicine, coordinated by the Institut Mérieux and supported by the French public agency BPI France. The VALIBI study group members are: Limoges: Marc Clavel, Nicolas Pichon, Philippe Vignon, Roselyne Droual, Cécile Duchiron, Julie Vignaud, Delphine Chainier. Brive: Elias Karam, Mathieu Mattei, André Sommabere. Tours: Emmanuelle Mercier, Cécile Le Brun. Angoulême: Arnaud Desachy, Caroline Garandeau. bioMérieux: Marc Rodrigue, Morgane Lacroix, Sandrine Prudent, Marie-Astrid Jestin.

\section{Compliance with ethical standards}

\section{Conflicts of interest}

$\mathrm{BF}, \mathrm{OB}$, and $\mathrm{MCP}$ institutions have received funding from bioMérieux to run the VALIBI study. JYM, Al, CAS, AP, and VM are employees of bioMérieux. S.A. CG is an employee of bioMérieux Inc. MR is an employee of BioFire Diagnostics LLC.

Accepted: 13 October 2018

Published online: 22 October 2018 


\section{References}

1. Wang Y, Eldridge N, Metersky ML, Verzier NR, Meehan TP, Pandolfi MM, Foody JM, Ho SY, Galusha D, Kliman RE, Sonnenfeld N, Krumholz HM, Battles J (2014) National trends in patient safety for four common conditions, 2005-2011. N Eng J Med 370:341-351

2. Ramanan P, Bryson AL, Binnicker MJ, Pritt BS, Patel R (2017) Syndromic panel-based testing in clinical microbiology. Clin Microbiol Rev 31:pii:e00024-17

3. Jamal W, Al Roomi E, AbdulAziz LR, Rotimi VO (2014) Evaluation of Curetis Unyvero, a multiplex PCR-based testing system, for rapid detection of bacteria and antibiotic resistance and impact of the assay on management of severe nosocomial pneumonia. J Clin Microbiol 52:2487-2492

4. Clavel M, Barraud O, Moucadel V, Meynier F, Karam E, Ploy MC, Francois B (2016) Molecular quantification of bacteria from respiratory samples in patients with suspected ventilator-associated pneumonia. Clin Microbiol Infect 22:812.e1-812.e7

5. Jourdain B, Joly-Guillou ML, Dombret MC, Calvat S, Trouillet JL, Gibert C, Chastre J (1997) Usefulness of quantitative cultures of BAL fluid for diagnosing nosocomial pneumonia in ventilated patients. Chest 111:411-418 